\title{
A Five-Sided Led-Cave Signal Synchronous Display System for Power Simulation System
}

\author{
Jihong KONG ${ }^{\mathrm{a}}$, Chao $\mathrm{WU}^{\mathrm{b}}$, Enhua QIU, ${ }^{\mathrm{a}}$, Man $\mathrm{HE}^{\mathrm{b}, 1}$, Yisong $\mathrm{LV}^{\mathrm{a}}$, and Yu Xuan \\ $\mathrm{WANG}^{\mathrm{b}}$ \\ ${ }^{a}$ CSG Power Generation Co., Ltd., Guangzhou, China \\ ${ }^{\mathrm{b}}$ MCC Wuhan Survey and Research Institute Co., Ltd., Wuhan, China
}

\begin{abstract}
In order to solve the problems of large rendering load and low frame rate caused by the large screen of the power system five-sided LED-CAVE immersive virtual simulation platform, the article proposes a $1+\mathrm{N}$ cluster rendering synchronization for the power system five-sided LED-CAVE Display management system. Configure the five-sided LED-CAVE with 1+N high-performance graphics rendering workstations and synchronous display cards according to the size and resolution of each screen. Through the synchronous rendering display system and 3D optical tracking and positioning system, achieve the effect that 5 screens refresh the stereo display simultaneously. This method improves the efficiency and immersive experience of power system virtual reality simulation rendering.
\end{abstract}

Keywords. Power simulation, virtual reality, LED-CAVE, cluster rendering, synchronous display

\section{Introduction}

The power system virtual reality simulation display provides a multi-faceted stereo display space through multi-channel synchronization technology, stereo display technology and three-dimensional space rendering algorithms. It allows employees to be immersed in a virtual simulation environment surrounded by three-dimensional images, thereby obtaining an immersive high-resolution 3D images and multi-degree of freedom interactive experience[1].

The power system virtual reality simulation CAVE display system generally has two modes: projection display and LED display. Multi-sided LED splicing screen display mode[2] is characterized by small footprint, flexible assembly, and simple maintenance. It is gradually replacing the projection display method. Although it is gradually replacing the projection display method, how to select the appropriate number of graphics rendering workstations according to the size of the screen[3], how to configure the relevant graphics card parameters, and how to synchronize the control of each graphics rendering workstation to accomplish the seamless splicing of pictures on the screen, as

\footnotetext{
${ }^{1}$ Corresponding Author, Man HE, MCC Wuhan Survey and Research Institute Co., Ltd., Wuhan 430000, China; E-mail: 329770684@qq.com.
} 
well as problems of heavy picture rendering load and low frame rate, still need to be further solved in practical applications.

\section{Scheme of System Construction}

In view of the problems existing in the existing technology, this paper proposes a solution for the signals synchronous display of five-sided LED-CAVE in power system. The $1+\mathrm{N}$ cluster rendering synchronous display management system[4] is used to synchronously control and stereoscopic display the five-sided LED-CAVE display screen. This method avoids the picture dislocation between the display screens on each side, improves the three-dimensional display effect, and enhances the user' $s$ immersive virtual reality experience.

\subsection{Technology Solution}

The $1+\mathrm{N}$ cluster rendering synchronous display management system assigns the rendering work carried out by the original workstation to $\mathrm{N}$ workstations, improves the rendering efficiency by cluster rendering, and then refreshes and transmits the rendering image at the same frequency through the synchronous card. Finally, it is spliced and displayed on the five-sided LED-CAVE screen as a complete picture, providing immersive stereoscopic rendering screen and space.

\subsection{Signal Synchronization Process}

The signal synchronization process is shown in figure 1 .

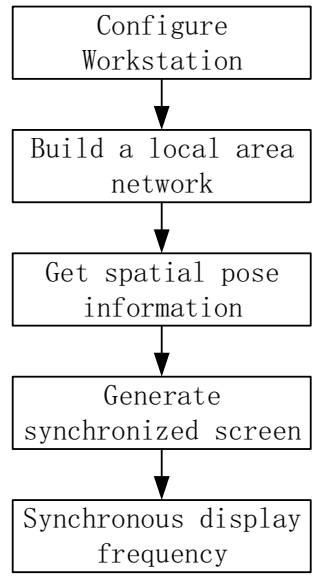

Figure 1. Diagram of Signal Synchronization Process.

Step one, configure the workstation. According to the size of the five-sided LEDCAVE display screen, the number of $1+\mathrm{N}$ high-performance graphics rendering workstations is configured. One of them is set as the main control computer, and the remaining $\mathrm{N}$ are allocated to five screens, namely, the front screen, the left screen, the right screen, the ground screen and the top screen according to the requirements. 
Second, build LAN. The synchronization cards of $1+\mathrm{N}$ high-performance graphics rendering workstations are connected in series through the network lines, and the master computer is connected with the host computer of the optical tracking and positioning system.

The third step, obtain the spatial pose information. The spatial position and attitude information of 3D glasses and operating handles are obtained by the optical positioning and tracking system and sent to the master machine.

The fourth step, generate synchronous screen[5]. The The host computer generates two sets of left-eye and right-eye images according to the spatial pose information of the $3 \mathrm{D}$ glasses and the operating handle, and sends them to other workstations. After the workstation finishes rendering, the images are displayed alternately on the LED screen.

The fifth step, synchronous display frequency. The main control machine controls the 3D signal transmitter to emit a control signal according to the frequency of the alternate display of the two sets of pictures and images, so that the switching display frequency of the two lenses of the 3D glasses is synchronized with the frequency of the alternate display of the two sets of pictures[6].

Through this process, a spatial (CAVE) stereo picture is generated between five LED splicing screens, allowing users to experience immersive virtual reality scenes.

\subsection{System Sharacteristics}

This five-side LED-CAVE signal synchronous display system for power system has the following characteristics :

- The five-sided LED-CAVE display consists of the front screen, the left screen, the right screen, the ground screen and the top screen;

- The signal synchronization system includes a five-sided LED-CAVE display, an optical positioning tracking system, and a $1+\mathrm{N}$ cluster rendering synchronization display management system;

- The $1+\mathrm{N}$ cluster rendering synchronization display management system includes a main control machine and $\mathrm{N}$ graphics rendering workstations and $3 \mathrm{D}$ signal transmitters connected to the main control machine;

- $\mathrm{N}$ graphics rendering workstations are used to control 5 LED-CAVE display screens;

- $\mathrm{N}$ graphics rendering workstations are configured with display and synchronization cards, which are serialized through the network;

- The master controller is connected to the host of optical positioning and tracking system via LAN;

- The master controller acts as a timing master node to control the synchronous output of $\mathrm{N}$ graphics rendering workstations; 


\section{Engineering Application Cases}

\subsection{Project Profile}

Southern Power Grid Peaking FM Power Generation Co., Ltd. independently developed a hydropower plant simulation platform based on immersive virtual reality technology [7], and built the five-sided LED-CAVE immersive maintenance simulation environment, which provides a simulation environment consistent with the real environment. It is applied to digital three-dimensional visualization of equipment, on-site auxiliary maintenance and decision-making, independent maintenance of operation and maintenance, safety emergency drills, staff training evaluation and skill competition, three-dimensional design and human-machine efficiency analysis.

\subsection{Implement Scheme}

According to the size and resolution of the five-sided LED-CAVE display screen in this project, seven high-performance graphics workstations are configured to form a $1+7$ cluster rendering synchronous display management system. The specific configuration is shown in Table 1, and the workstation allocation is shown in figure 2.

Table 1. Screen Size and Configuration

\begin{tabular}{cccccc}
\hline Screen Part & $\begin{array}{c}\text { Screen } \\
\text { Size }(\mathbf{m m})\end{array}$ & $\begin{array}{c}\text { Screen } \\
\text { Resolution }\end{array}$ & $\begin{array}{c}\text { Workstation } \\
\text { Quantity }\end{array}$ & $\begin{array}{c}\text { Workstation } \\
\text { Model }\end{array}$ & Serial Number \\
\hline left & $2400 * 2700$ & $1536 * 1728$ & 1 & HPZ840 & 01 \\
front & $4200 * 2700$ & $2688 * 1728$ & 2 & HPZ840 & $02 、 03$ \\
right & $2400 * 2700$ & $1536 * 1728$ & 1 & HPZ840 & 04 \\
ground & $4500 * 2500$ & $1536 * 1728$ & 1 & HPZ840 & 05 \\
top & $4200 * 2362.5$ & $2688 * 1512$ & 2 & HPZ840 & $06 、 07$ \\
\hline
\end{tabular}

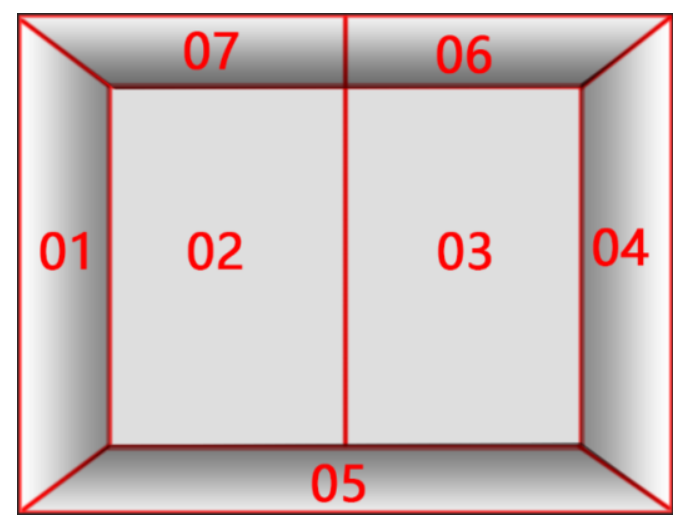

Figure 2. Diagram of Workstation Distribution

1+7 graphics rendering workstations are configured with a display card and a synchronization card. Synchronous cards are connected in series to control the display of five LED-CAVE screens. As shown in figure 3, two signal ports on the master 
synchronization card are used as the output ports, and the remaining synchronization cards are connected by net wire in series.

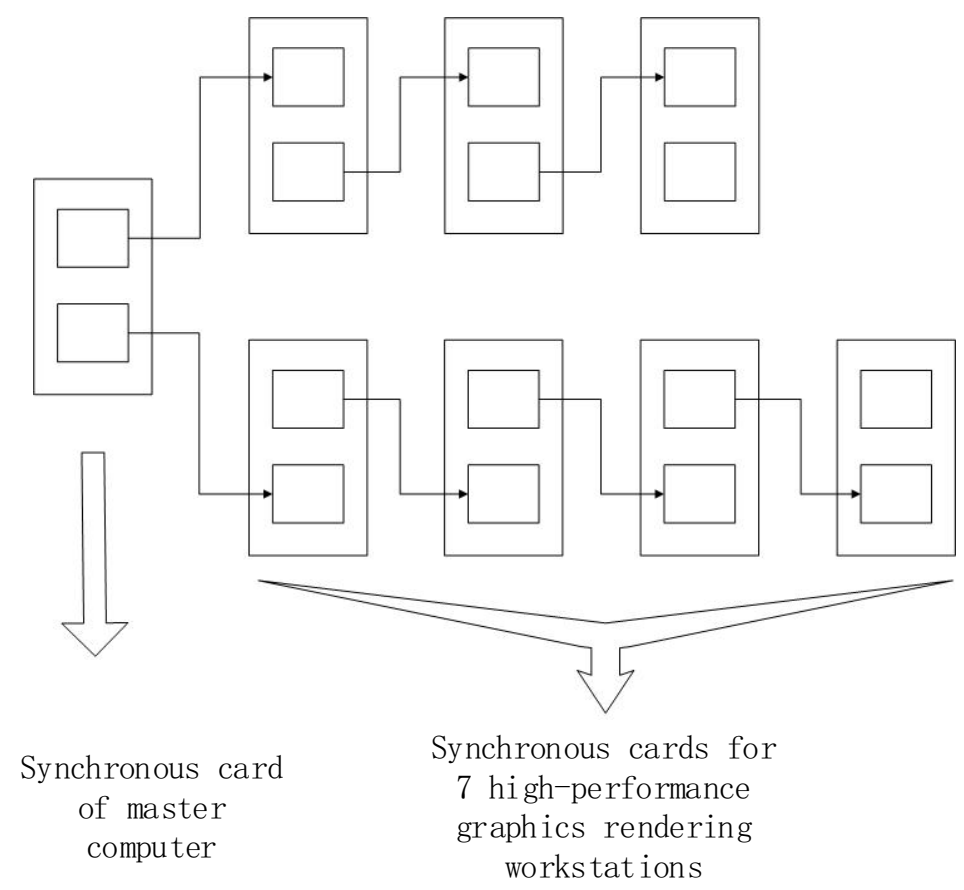

Figure 3. Diagram of Synchronous Card Series Connection

In this project, in order to ensure the effect of 3D display and avoid image jitter, the image refresh rate is set to $96 \mathrm{~Hz}[7]$, that is, the refresh rate of 7 graphics rendering workstations is $96 \mathrm{~Hz}$ according to the display card, and the refresh rate of left and right glasses is $48 \mathrm{~Hz}$.

The master computer and the host computer of the optical positioning and tracking system are connected through the local area network. The optical positioning and tracking system generates spatial pose information through the positioning ball on the $3 \mathrm{D}$ glasses and transmits it to the master computer. According to these information, the master computer generates two sets of images of the left eye and the right eye, and synchronously outputs the signal to seven graphics rendering workstations. The final picture is alternately displayed in the CAVE immersive simulation platform formed by five screens according to the refresh frequency. The 3D glasses worn by the operator switch the left and right eyes at the same frequency, so as to achieve the immersive virtual reality effect.

The overall system architecture is shown in figure 4. 


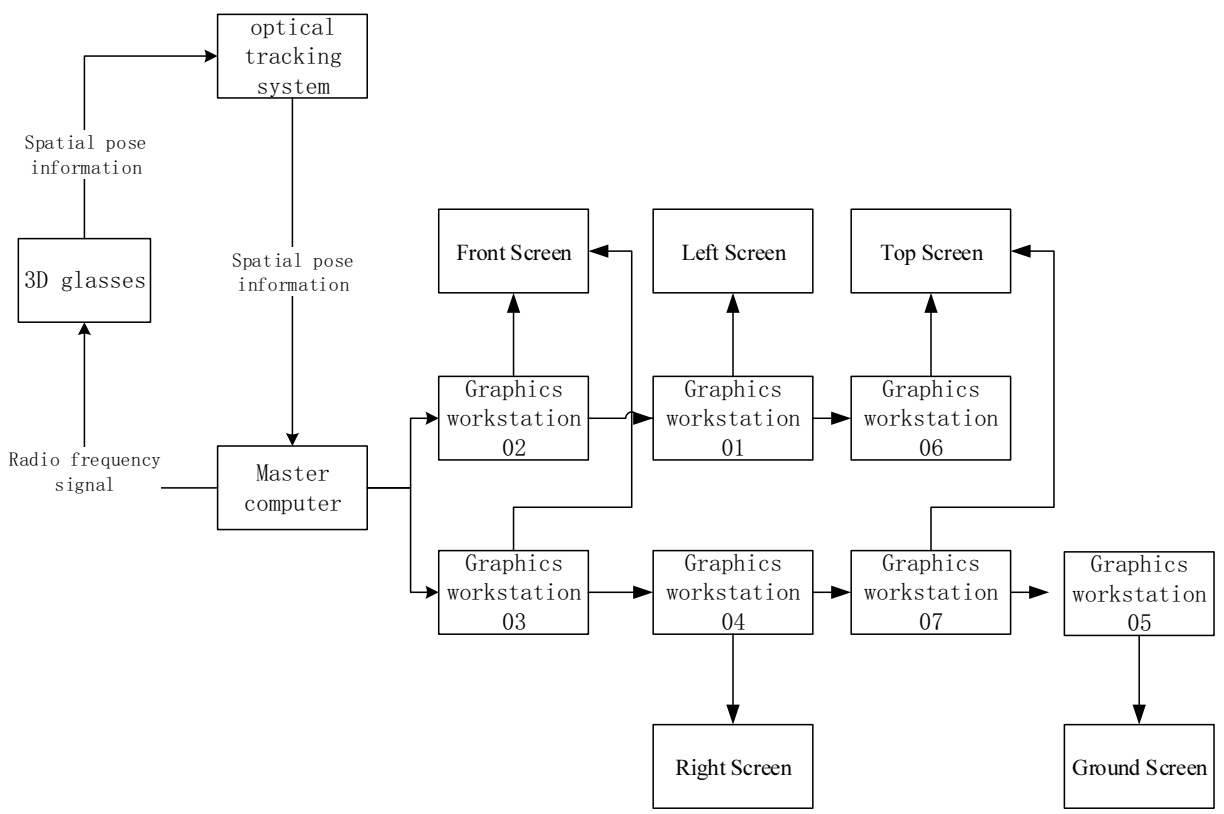

Figure 4. Diagram of Overall Structure

\subsection{Application}

The virtual reality immersive simulation platform of power system can provide a simulation environment consistent with the operation and maintenance environment of power plant. In this environment, the staff can not only carry out normal operation training, but also drill various accidents and fault handling. The simulation platform is of great significance to improve the operation skills and level of power plant staff, enhance the strain processing ability, avoid equipment damage caused by operating errors, power grid splitting and other major accidents. The results of the project have been applied in large-scale practice in the fields of auxiliary maintenance of hydropower plants, digital and accurate modeling of equipment[8], independent maintenance of operation and maintenance, safety emergency drill, practical operation of skill competition, training evaluation, three-dimensional design and human-machine efficacy analysis. The actual application effect is shown in figure 5 . 


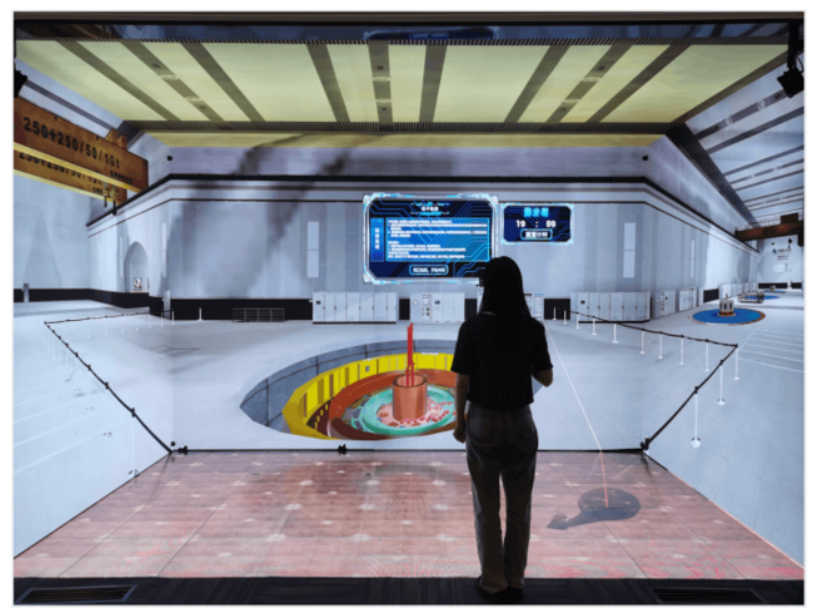

Figure 5. Diagram of Running Result

\section{Conclusion}

$1+\mathrm{N}$ cluster rendering synchronous display management system solved the problems of large rendering load and low frame rate caused by single workstation control in power system five-sided LED-CAVE display system. Cluster rendering system assigns the rendering work of one workstation to $\mathrm{N}$ workstations at the same time, and improves the rendering frame rate by reducing the load of a single workstation. The synchronous display system reduces the delay of signal transmission by serial connection of synchronous cards, so that the pictures rendered by each graphics rendering workstation can be refreshed and displayed synchronously, making $\mathrm{N}$ groups of pictures look like a whole image. this method improving the immersion and use experience of power system simulation.

\section{References}

[1] Zhao Meng. Large-Screen Multi-Person Immersive Stereo Display System[D]. Dalian Maritime University, 2010.

[2] Qiu Enhua, Kong Jihong, Zhou Gang, Ji Huaijie, Zhang Fangming, Wu Chao, Chen Shikun. A Structural Device For Adjusting The Flatness Of Led Display Screens[J]. Mechatronic Engineering Technology,2019,48(12):140-142.

[3] Bi Wenqiang.The Hardware Development Trend Of Graphics Workstations And Its Impact On The Radio And Television Industry [J] .Modern Television Technology, 2014 (10): 48-50.

[4] Fan Danyang. Research on Multi-channel Visual Simulation System Based on PC Cluster [D]. Xi' an University of Architecture and Technology, 2014.

[5] Bao Rong.High-Precision Positioning With Laser Tracking And Ranging System[J].Laser and Infrared,1979(08):1-5.

[6] Zhu Xianghai. Design And Implementation Of 3D Glasses Based On Radio Frequency Communication[D]. Hangzhou Dianzi University, 2016.

[7] Li Xilin.Talking About The Refresh Frequency And Frame Changing Frequency Of Led Large Screen[J].Modern Display,2004(01):22-26.

[8] Kong Jihong,Wu Chao,Qiu Enhua,He Man,Lv Yisong. Automatically Trimming Profile Based On Triangular Mesh Model[J]. Journal of Physics: Conference Series,2021,1732(1): 